\title{
New results on collectivity in small systems with ATLAS
}

\author{
Mariusz Przybycien ${ }^{* \dagger}$ (on behalf of the ATLAS Collaboration) \\ AGH University of Science and Technology \\ Faculty of Physics and Applied Computer Science \\ Al. Mickiewicza 30, 30-059 Cracow, Poland \\ E-mail: mariusz.przybycien@agh.edu.pl
}

\begin{abstract}
The azimuthal anisotropies of particle yields observed in relativistic heavy-ion collisions have been traditionally considered as a strong evidence of the formation of a deconfined quark-gluon plasma produced in these collisions. However multiple recent measurements from the LHC experiments in $p p$ and $p+\mathrm{Pb}$ systems show similar features as those observed in $\mathrm{Pb}+\mathrm{Pb}$ collisions, indicating the possibility of the production of such a deconfined medium in smaller collision systems. This report presents a comprehensive summary of ATLAS measurements in $p p$ collisions at 2.76, 5.02 and $13 \mathrm{TeV}$ and in $p+\mathrm{Pb}$ collisions at 5.02 and $8.16 \mathrm{TeV}$. It includes measurements of two-particle hadron-hadron correlations in $\Delta \phi$ and $\Delta \eta$, with a template fitting procedure used to subtract the dijet contributions. Measurements of multi-particle cumulants $c_{n}\{2-8\}$ are also presented. The standard cumulant measurements confirm presence of collective phenomena in $p+\mathrm{Pb}$ collisions, but are biased by non-flow correlations and are not able to provide evidence for collectivity in $p p$ collisions. To address this, measurements from a new sub-event cumulant method that suppresses the contribution of non-flow effects are discussed. Also the first measurements of $\mathrm{v}_{2}$ coefficient in $Z$-tagged $p p$ events and with $D^{\star \pm}$ mesons in the final state in $p+\mathrm{Pb}$ collisions are presented.
\end{abstract}

Sixth Annual Conference on Large Hadron Collider Physics (LHCP2018)

4-9 June 2018

Bologna, Italy

\footnotetext{
* Speaker.

$\dagger$ This work was partly supported by the National Science Centre of Poland under grant number UMO2016/23/B/ST2/01409, by the AGH UST statutory tasks No. 11.11.220.01/4 within subsidy of the Ministry of Science and Higher Education and by PL-GRID infrastructure.
} 


\section{Introduction}

Measurements of azimuthal correlations in small collision systems, such as $p p$ or $p+\mathrm{A}$ collisions, have revealed the ridge phenomenon [1,2,3], a feature first observed in $\mathrm{A}+\mathrm{A}$ collisions: enhanced production of particle pairs at small azimuthal angle separation, $\Delta \phi$, extended over a wide range of pseudorapidity separation, $\Delta \eta$. The ridge reflects multi-parton dynamics early in the collision and has generated significant interest in the high-energy physics community. A key question is whether the long-range multi-particle collectivity reflects initial momentum correlation from gluon saturation effects [4], or a final-state hydrodynamic response to the initial collision geometry [5].

The ridge signature from the two-particle (2PC) angular correlations is characterized by a Fourier decomposition of the correlation function $C(\Delta \phi) \sim 1+2 \sum_{n} \mathrm{v}_{n}^{2} \cos (n \Delta \phi)$, where $\mathrm{v}_{n}$ are the single-particle anisotropy harmonic coefficients. An important feature of the ridge is whether it involves all particles in the event (collective flow) or if it arises merely from correlations among a few particles, due to resonance decays, jets, or multijet production (non-flow). In small systems the contributions from non-flow sources, in particular from jets and dijets, are large. Since collective flow is intrinsically a multiparticle phenomenon, it can be probed more directly using cumulants based on multiparticle correlation techniques. The standard cumulant methods involve the calculation of $2 k$-particle azimuthal correlations $\left\langle\{2 k\}_{n}\right\rangle$, and $2 k$-particle cumulants, $c_{n}\{2 k\}$, for the $n$ th-order flow harmonics. For example, the two- and four-particle cumulants are obtained from the azimuthal correlations, and the flow coefficients are then defined as:

$c_{n}\{2\}=\left\langle\left\langle\{2\}_{n}\right\rangle\right\rangle, c_{n}\{4\}=\left\langle\left\langle\{4\}_{n}\right\rangle\right\rangle-2\left\langle\left\langle\{2\}_{m}\right\rangle\right\rangle^{2} \Rightarrow \mathrm{v}_{n}\{2\}=\sqrt{c_{n}\{2\}}, \mathrm{v}_{n}\{4\}=\sqrt[4]{-c_{n}\{4\}}$

where $\langle\langle\rangle\rangle$ represents the average of $\left\langle\{2 k\}_{n}\right\rangle$ over an event ensemble. In the standard cumulant method described above, all $2 k$-particle multiplets involved in $\left\langle\{2 k\}_{n}\right\rangle$ are selected using the entire detector acceptance. To further suppress the non-flow correlations that typically involve particles emitted within a localized region in $\eta$, the particles can be grouped into several subevents, each covering a non overlapping $\eta$ interval. The multiparticle correlations are then constructed by correlating particles between different subevents, further reducing non-flow correlations. For example, in the two-subevent cumulant method (2SE), the entire event is divided into two subevents, labelled as $a$ and $b$, according to $-\eta_{\max }<\eta_{a}<0$ and $0<\eta_{b}<\eta_{\max }$, where $\eta_{\max }=2.5$ is the maximum $\eta$ used in the analysis and corresponds to the ATLAS detector acceptance for charged particles. Multi-particle correlations are constructed by correlating tracks from different subevents, e.g.:

$$
\begin{aligned}
& \left\langle\{2\}_{n}\right\rangle_{a \mid b}=\left\langle e^{i n\left(\phi_{1}^{a}-\phi_{2}^{b}\right)}\right\rangle, \quad\left\langle\{3\}_{n}\right\rangle_{2 a \mid b}=\left\langle e^{i n\left(\phi_{1}^{a}+\phi_{2}^{a}-2 \phi_{3}^{b}\right)}\right\rangle, \quad\left\langle\{4\}_{n, m}\right\rangle_{2 a \mid 2 b}=\left\langle e^{i n\left(\phi_{1}^{a}-\phi_{2}^{b}\right)+i m\left(\phi_{3}^{a}-\phi_{4}^{b}\right)}\right\rangle \\
& c_{n}^{a \mid b}\{2\}=\left\langle\left\langle\{2\}_{n}\right\rangle\right\rangle_{a \mid b}, \quad c_{n}^{2 a \mid 2 b}\{4\}=\left\langle\left\langle\{4\}_{n}\right\rangle\right\rangle_{2 a \mid 2 b}-2\left\langle\left\langle\{2\}_{n}\right\rangle\right\rangle_{a \mid b}^{2}
\end{aligned}
$$

In case of three- and four-subevent cumulant methods the available pseudorapidity range is divided into three or four equidistant subevents, respectively. In general, the 2SE method allows to remove most of intra-jet correlations, the 3SE method removes most of inter-jet correlations and the 4SE method removes partly also inter-jet correlations in the jets belonging to two adjacent subevents.

The multi-particle cumulants probe the event-by-event fluctuation of a single flow harmonic $\mathrm{v}_{n}$, as well as the correlated fluctuations between two flow harmonics, $\mathrm{v}_{n}$ and $\mathrm{v}_{m}$. For instance, the 
four-particle symmetric cumulants $\mathrm{sc}_{n, m}\{4\}=\left\langle\mathrm{v}_{n}^{2} \mathrm{v}_{m}^{2}\right\rangle-\left\langle\mathrm{v}_{n}^{2}\right\rangle\left\langle\mathrm{v}_{m}^{2}\right\rangle$ quantify the lowest-order correlation between $\mathrm{v}_{n}$ and $\mathrm{v}_{m}$. The three-particle asymmetric cumulant $\operatorname{ac}_{n}\{3\}=\mathrm{v}_{n}^{2} \mathrm{v}_{2 n} \cos 2 n\left(\Phi_{n}-\Phi_{2 n}\right)$ is sensitive to correlations involving both the flow magnitude $\mathrm{v}_{n}$ and flow phase $\Phi_{n}$.

In the next section a selection of recent results on collectivity in small systems, obtained from the ATLAS [6] experiment at the LHC is briefly discussed.

\section{Selected results on collectivity in small systems}

Figure 1 presents direct comparisons of the $c_{2}\{4\}$ cumulant obtained from the standard, 2SE and 3SE methods for $p p$ and $p+\mathrm{Pb}$ collisions at $\sqrt{s_{\mathrm{NN}}}=5.02 \mathrm{TeV}$ as a function of average number of charged particles in the event, $\left\langle N_{\mathrm{ch}}\right\rangle$, [7]. In $p p$ collisions the $c_{2}\{4\}$ values are smallest for the 3SE method and largest for the standard method. The same hierarchy between the three methods is also observed in $p+\mathrm{Pb}$ collisions, but only for the $\left\langle N_{\mathrm{ch}}\right\rangle<100$ region, suggesting that non-flow effects in $p+\mathrm{Pb}$ collisions are much smaller than those in $p p$ collisions at comparable $\left\langle N_{\mathrm{ch}}\right\rangle$. In $p+\mathrm{Pb}$ collisions, all three methods give consistent results for $\left\langle N_{\mathrm{ch}}\right\rangle>100$. Furthermore, the 3SE method gives negative $c_{2}\{4\}$ values (as they should be) in most of the measured $\left\langle N_{\mathrm{ch}}\right\rangle$ range.

The correlations between flow harmonics have been studied by ATLAS via symmetric and asymmetric cumulants. The measurements of $\operatorname{sc}_{2,3}\{4\}, \mathrm{sc}_{2,4}\{4\}$ and $\mathrm{ac}_{2}\{3\}$ have been performed in $p p$ collisions at $\sqrt{s}=13 \mathrm{TeV}, p+\mathrm{Pb}$ collisions at $\sqrt{s_{\mathrm{NN}}}=5.02 \mathrm{TeV}$ and low-multiplicity $\mathrm{Pb}+\mathrm{Pb}$ collisions at $\sqrt{s_{\mathrm{NN}}}=2.76 \mathrm{TeV}$ [8]. They are obtained using 2SE, 3SE and 4SE cumulant methods and are compared with results from the standard cumulant method. The results of the measurements are shown in Fig. 2. Significant differences are observed between the standard method and the subevent methods over the full $\left\langle N_{\mathrm{ch}}\right\rangle$ range in $p p$ collisions, as well as over the low $\left\langle N_{\mathrm{ch}}\right\rangle$ range in $p+\mathrm{Pb}$ and $\mathrm{Pb}+\mathrm{Pb}$ collisions. The differences are larger for particles at higher $p_{\mathrm{T}}$ (see Ref. [8]) or at smaller $\left\langle N_{\mathrm{ch}}\right\rangle$. When analysed with the standard method in $p p$ collisions, this behaviour is compatible with the dominance of the non-flow correlations rather than the long-range collective flow correlations. Systematic, but much smaller, differences are also observed in the low $\left\langle N_{\mathrm{ch}}\right\rangle$ region between the 2SE method and 3SE or 4SE methods, which indicate that the 2SE method may still be affected by correlations arising from jets. On the other hand no differences are observed between the 3SE and 4SE methods, within experimental uncertainties, suggesting that methods
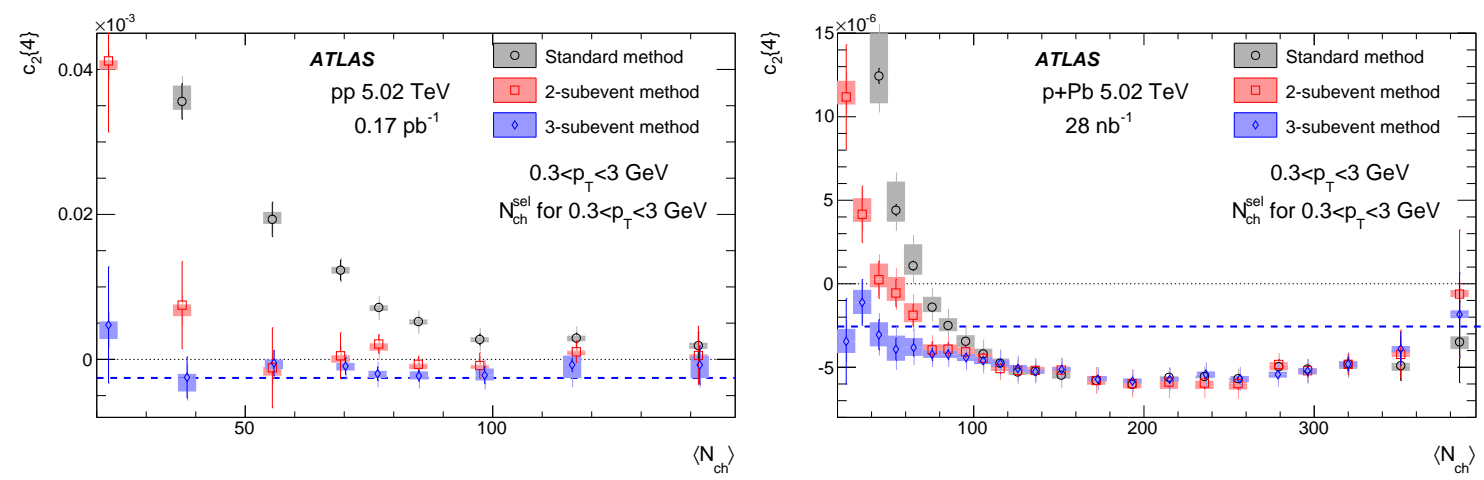

Figure 1: The $c_{2}\{4\}$ values calculated for charged particles with $0.3<p_{\mathrm{T}}<3 \mathrm{GeV}$ compared for the three cumulant methods from the $5.02 \mathrm{TeV} p p$ (left) and $p+\mathrm{Pb}$ (right) data [7]. The error bars and shaded boxes represent the statistical and systematic uncertainties, respectively. 

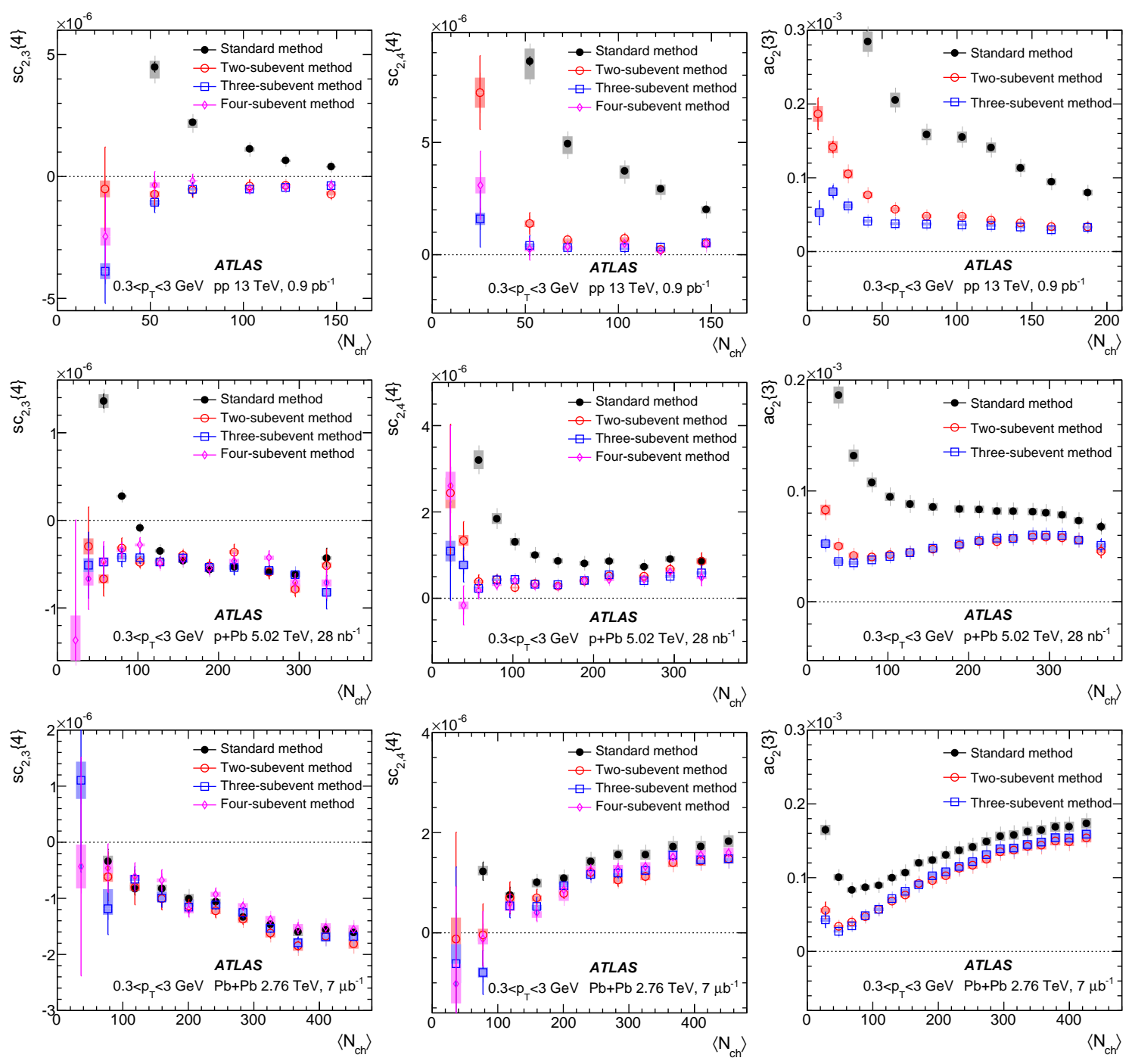

Figure 2: The symmetric cumulants $\mathrm{sc}_{2,3}\{4\}$ (left) and $\mathrm{sc}_{2,4}\{4\}$ (middle) and the asymmetric cumulant $\mathrm{ac}_{2}\{3\}$ (right) as a function of $\left\langle N_{\mathrm{ch}}\right\rangle$ for $0.3<p_{\mathrm{T}}<3 \mathrm{GeV}$ obtained for $p p$ collisions (top row), $p+\mathrm{Pb}$ collisions (middle row) and low-multiplicity $\mathrm{Pb}+\mathrm{Pb}$ collisions (bottom row) [8]. The symmetric cumulants are obtained from the standard method (filled symbol), the 2SE method (open circles), the 3SE method (open squares) and the 4SE method (open diamonds). The asymmetric cumulant is obtained from the standard method (filled symbol), the 2SE method (open circles), and the 3SE method (open squares). The error bars and shaded boxes represent the statistical and systematic uncertainties, respectively.

with three or more subevents are sufficient to reject non-flow correlations from jets. Therefore, the 3SE method is used to present the main results in this analysis. Figure 3 shows a direct comparison of cumulants for the three collision systems. The three panels in the top row show the results for $\operatorname{sc}_{2,3}\{4\}, \operatorname{sc}_{2,4}\{4\}$ and $\operatorname{ac}_{2}\{3\}$, respectively. These results support the existence of a negative correlation between $\mathrm{v}_{2}$ and $\mathrm{v}_{3}$ and a positive correlation between $\mathrm{v}_{2}$ and $\mathrm{v}_{4}$. Such correlation patterns have previously been observed in large collision systems, but are now confirmed also in the small collision systems, once non-flow effects are adequately suppressed. In the multiplicity 

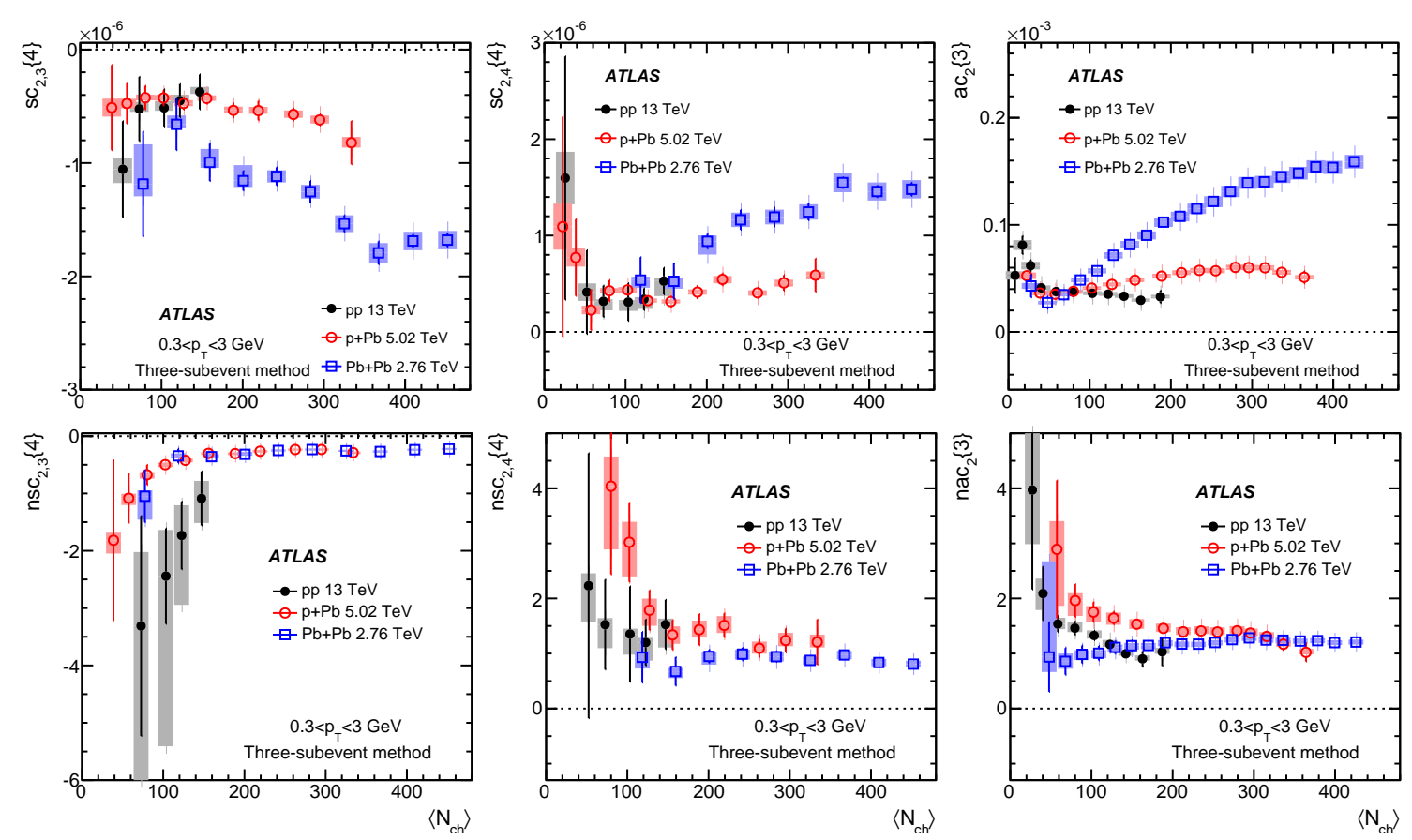

Figure 3: The $\left\langle N_{\mathrm{ch}}\right\rangle$ dependence of $\operatorname{sc}_{2,3}\{4\}, \mathrm{sc}_{2,4}\{4\}$ and $\operatorname{ac}_{2}\{3\}$ (top row) and $\mathrm{nsc}_{2,3}\{4\}, \mathrm{nsc}_{2,4}\{4\}$ and nac $_{2}\{3\}$ (bottom row) in $0.3<p_{\mathrm{T}}<3 \mathrm{GeV}$ obtained for $p p$ collisions (solid circles), $p+\mathrm{Pb}$ collisions (open circles) and low-multiplicity $\mathrm{Pb}+\mathrm{Pb}$ collisions (open squares) [8]. The error bars and shaded boxes represent the statistical and systematic uncertainties, respectively.

range covered by the $p p$ collisions, $\left\langle N_{\mathrm{ch}}\right\rangle<150$, the results for symmetric cumulants $\mathrm{sc}_{2,3}\{4\}$ and $\operatorname{sc}_{2,4}\{4\}$ are similar among the three systems. In the range $\left\langle N_{\mathrm{ch}}\right\rangle>150, \mathrm{sc}_{2,3}\{4\}$ and $\operatorname{sc}_{2,4}\{4\}$ are larger in $\mathrm{Pb}+\mathrm{Pb}$ than in $p+\mathrm{Pb}$ collisions. The results for $\mathrm{ac}_{2}\{3\}$ are similar among the three systems at $\left\langle N_{\mathrm{ch}}\right\rangle<100$, but they deviate from each other at higher $\left\langle N_{\mathrm{ch}}\right\rangle$. The $p p$ data are approximately constant or decrease slightly with $\left\langle N_{\mathrm{ch}}\right\rangle$, while the $p+\mathrm{Pb}$ and $\mathrm{Pb}+\mathrm{Pb}$ data show significant increases as a function of $\left\langle N_{\mathrm{ch}}\right\rangle$. The cumulants are then normalised by the $\left\langle\mathrm{v}_{n}^{2}\right\rangle$ obtained from a two-particle correlation analysis [9] to quantify their relative correlation strength. In the bottom row of Fig. 3 the results for normalised cumulants, $\operatorname{nsc}_{2,3}\{4\}, \operatorname{nsc}_{2,4}\{4\}$ and $\operatorname{nac}_{2}\{3\}$ are compared among the three systems. The normalised cumulants generally show a much weaker $\left\langle N_{\mathrm{ch}}\right\rangle$ dependence at $\left\langle N_{\mathrm{ch}}\right\rangle>100$, where the statistical uncertainties are small. This behaviour implies that the strong $\left\langle N_{\mathrm{ch}}\right\rangle$ dependence of the $\operatorname{sc}_{n, m}\{4\}$ and $\operatorname{ac}_{2}\{3\}$ values reflects the $\left\langle N_{\mathrm{ch}}\right\rangle$ dependence of the $\mathrm{v}_{n}$ values, and these dependences are removed in the normalised cumulants. The normalised cumulants are also similar among different collision systems at large $\left\langle N_{\mathrm{ch}}\right\rangle$, although some differences at the relative level of $20-30 \%$ are observed for smaller $\left\langle N_{\mathrm{ch}}\right\rangle$. The only exception is $\mathrm{nsc}_{2,3}\{4\}$, whose values in the $p p$ collisions are very different from those in $p+\mathrm{Pb}$ and $\mathrm{Pb}+\mathrm{Pb}$ collisions. In contrast, the $\operatorname{sc}_{2,3}\{4\}$ values are close among different systems. This suggests that the $\left\langle v_{3}^{2}\right\rangle$ values from the template fit method may be significantly underestimated. As pointed out in Ref. [9], the template fit method, and other methods based on peripheral subtraction in general, tend to underestimate the odd flow harmonics, due to the presence of a large away-side peak at $\Delta \phi \sim \pi$ in the 2PC correlation function. The comparison of $\operatorname{sc}_{2,3}\{4\}$ and $\operatorname{nsc}_{2,3}\{4\}$ among different collision systems provides indirect evidence of this underestimation of $\left\langle v_{3}^{2}\right\rangle$. 

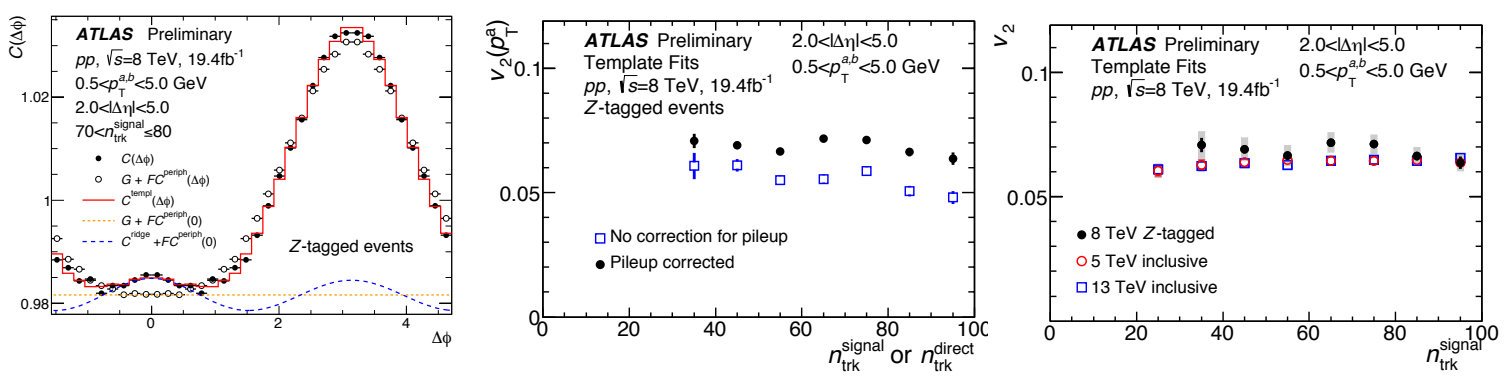

Figure 4: (left) Template fits to the pileup corrected $C(\Delta \phi)$. (middle) The $\mathrm{v}_{2}$ values obtained from the template fits with and without correcting for pileup pairs. (right) The $v_{2}$ values corrected for pileup compared to the $\mathrm{v}_{2}$ obtained in $13 \mathrm{TeV}$ and $5 \mathrm{TeV}$ inclusive $p p$ data, [10].

ATLAS performed first study of long-range hadron correlations in $p p$ collisions with a constraint on collision geometry, by requiring a $Z$ boson in the final state [10]. The presence of a $Z$ boson selects an event in which there was a hard scattering (with $Q^{2} \approx 80 \mathrm{GeV}^{2}$ ), which may lower the partonic impact parameter $b$. An assumption driven by the $\mathrm{A}+\mathrm{A}$ collisions is that if the $p p \mathrm{v}_{2}$ is related to the eccentricity of the collision geometry, then events 'tagged' by a $Z$ boson having a smaller $b$ might also have smaller $\mathrm{v}_{2}$ than that measured in inclusive events. The data used in this analysis comes from high luminosity $p p$ running at $\sqrt{s}=8 \mathrm{TeV}$, when pileup was at the level $\mu \approx 20$. To solve the problem of pileup tracks a new procedure was developed to remove the contribution of such tracks on a statistical basis. The result of the template fit method applied to pileup corrected $Z$ boson tagged 2PC function $C(\Delta \phi)$ is shown in Fig. 4 (left). In Fig. 4 (middle) the $v_{2}$ obtained from the template fit with and without pileup subtraction is shown as the number of charged tracks. The right panel of Fig. 4 shows the final $\mathrm{v}_{2}$ values obtained from the template fit in the $8 \mathrm{TeV} Z$-tagged sample, compared to the $\mathrm{v}_{2}$ values obtained in $13 \mathrm{TeV}$ and $5 \mathrm{TeV}$ inclusive $p p$ collisions from Ref. [9]. The $Z$-tagged $8 \mathrm{TeV}_{2}$ values show at most a weak dependence on the multiplicity, similar to the results obtained with the inclusive samples. However, the $Z$-tagged $\mathrm{v}_{2}$ values are slightly larger than the inclusive $\mathrm{v}_{2}$ results. This difference is likely to be not due to the difference in $\sqrt{s}$, as both the 5 and $13 \mathrm{TeV}$ inclusive $\mathrm{v}_{2}$ results are consistent with each other, implying an $\sqrt{s}$ independence of the $p p \mathrm{v}_{2}$.

Heavy quarks are primarily produced at early stages of heavy-ion collisions due to their large mass, and could carry information about the early stage properties of the quark-gluon plasma (QGP) created in such collisions. Compared with gluons and light quarks, heavy quarks are expected to lose less energy when traversing the medium. ATLAS has studied production yields of $D^{\star \pm}$ mesons and azimuthal angular correlations between charged particles and inclusive $D^{\star}$ candidates in $p+\mathrm{Pb}$ collisions at $\sqrt{s_{\mathrm{NN}}}=8.16 \mathrm{TeV}$ [11]. The resulting $D^{\star}-$ hadron $\left(D^{\star}-h\right)$ correlation functions with template fits are shown in Fig. 5 in three multiplicity intervals. In events with low multiplicity, $10<N_{\mathrm{ch}}<80$, the $D^{\star}-h$ correlation function is consistent with being flat at $\Delta \phi \sim 0$ which indicates a near-side ridge with a small amplitude. In events with $N_{\mathrm{ch}}>80$, the coefficient for $\cos (2 \Delta \phi)$ modulation, $\mathrm{v}_{2,2} \equiv \mathrm{v}_{2}^{2}$, for the $D^{\star}-h$ correlation are measured to be different from zero and their values are displayed in the plots on Fig. 5 . 

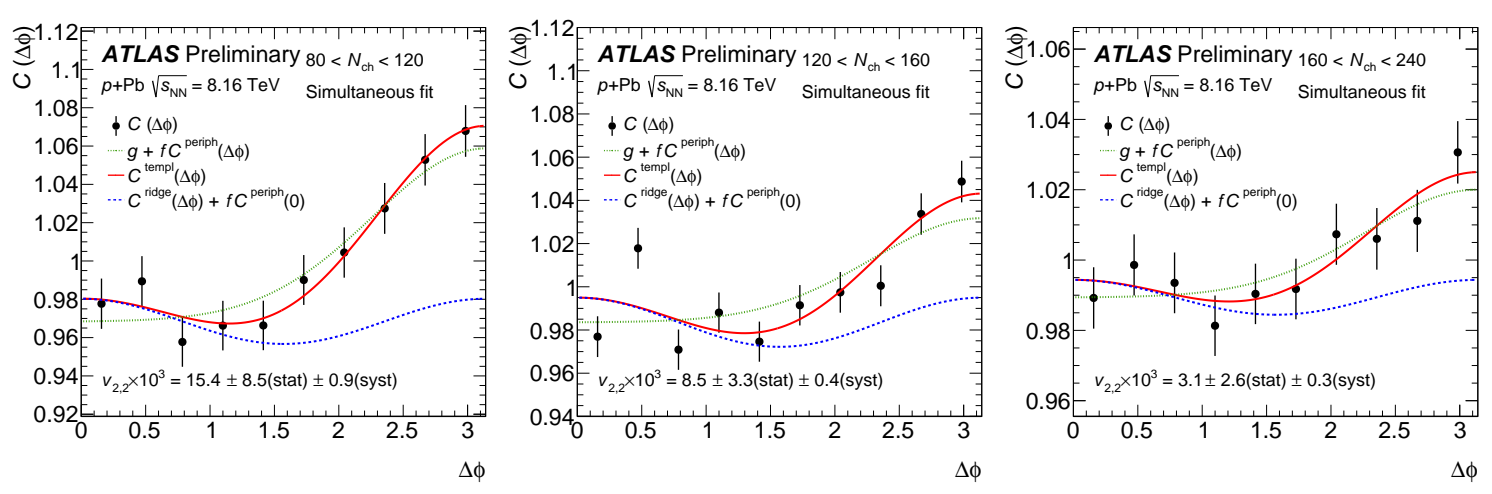

Figure 5: Template fits to the $D^{\star}-h$ correlation function for different multiplicity intervals. Fit results for different multiplicity events from simultaneous fits to the low multiplicity range $(10-80)$ and higher multiplicity correlation functions (the ranges are displayed in the plots) are plotted as lines. The resulting $\mathrm{v}_{2,2}$ values and their uncertainties are also shown, [11].

\section{Summary}

The subevent cumulant technique and the new results provide direct evidence that the ridge is indeed a long-range collective phenomenon involving many particles distributed across a broad rapidity interval. The results of $c_{2}\{4\}$ as well as on symmetric and asymmetric cumulants and their dependence on $\left\langle N_{\mathrm{ch}}\right\rangle$ and $p_{\mathrm{T}}$, largely free from nonflow effects, can be used to understand the spacetime dynamics and the properties of the medium created in small collision systems. These results, available now for $p p, p+\mathrm{Pb}$ and $\mathrm{Pb}+\mathrm{Pb}$ collisions, provide inputs to distinguish between models based on initial-state momentum correlations and models based on final-state hydrodynamics.

First measurements of the $\mathrm{v}_{2}$ coefficients in $Z$ boson tagged $p p$ collisions and in $p+\mathrm{Pb}$ collisions with $D^{\star}$ meson in the final state have been also performed. The $\mathrm{v}_{2}$ coefficients for inclusive $D^{\star}$ and charged particles azimuthal correlation have been extracted using template fits based on $p+\mathrm{Pb}$ collisions at $\sqrt{s_{\mathrm{NN}}}=8.16 \mathrm{TeV}$.

\section{References}

[1] CMS Collaboration, J. High Energy Phys. 09 (2010) 091, arXiv:1009.4122 [hep-ex].

[2] ALICE Collaboration, Phys. Lett. B 719 (2013) 29, arXiv:1212.2001 [nucl-ex].

[3] ATLAS Collaboration, Phys. Rev. Lett. 110 (2013) 182302, arXiv:1212.5198 [hep-ex].

[4] K. Dusling, R. Venugopalan, Phys. Rev. D 87 (2013) 094034, arXiv:1302.7018 [hep-ph].

[5] P. Bozek, W. Broniowski, Phys. Rev. C 88 (2013) 014903, arXiv:1304.3044 [nucl-th].

[6] ATLAS Collaboration, JINST 3 (2008) S08003.

[7] ATLAS Collaboration, Phys. Rev. C 97 (2018) 024904, arXiv:1708.03559 [nucl-ex].

[8] ATLAS Collaboration, arXiv:1807.02012 [nucl-ex].

[9] ATLAS Collaboration, Phys. Rev. C 96 (2017) 024908, arXiv:1609.06213 [nucl-ex].

[10] ATLAS Collaboration, ATLAS-CONF-2017-068.

[11] ATLAS Collaboration, ATLAS-CONF-2017-073. 